\title{
Tiempo de jugar, tiempo de morir: Apuntes sobre Until Dawn y Man of Medan (Supermassive Games, 2015 y 2019)
}

Aaron Rodríguez Serrano | serranoa@uji.es Universitat Jaume I, Castellón (España)

\section{Resumen}

Palabras clave

"Man of Medan"; "Muerte"; "Narratología";

"Until Dawn"; "Videojuegos".

Sumario

1.Introducción: La estructura y la serie.

1.1 La muerte como clausura del relato.

1.2 Morir en un videojuego.

2. Supermassive Games: El mal y la muerte.

2.1 El mal teológico: Sobre los narradores.

2.2 Del mal de los hombres al mal de los

dioses.

2.3 De cómo escapar a la muerte:

Herramientas cognitivas.

2.4 De cómo pensar la muerte: Herramientas simbólicas.

3. Conclusiones.

4. Bibliografía.
El presente artículo pretende realizar una aproximación desde el análisis textual a los juegos Until Dawn y Man of Medan (Supermassive Games, 2015 y 2019), partiendo de la problemática que supone el concepto de "muerte" en el videojuego. Para ello, se analizarán ambos juegos en torno a los siguientes factores: en primer lugar, se realizará un breve análisis de lo que implica morir en el videojuego en relación con las teorías propias de la clausura textual - el llamado "modo del relato clásico" y su relación con el sentido de los textos. En segundo lugar, se procederá al análisis propio de los juegos, partiendo de dos directrices: la naturaleza del mal -en sus dimensiones humana (esencialista) y sobrenatural (literaria)-, y la posición que ambos juegos toman sobre la muerte -en cuanto a mecánicas y recursos cognitivos, pero también en cuanto a su dimensión simbólica. Finalmente, se ofrecen las conclusiones y se plantean algunas sugerencias con respecto al futuro de la investigación.

\section{Cómo citar este texto:}

Aarón Rodríguez Serrano (2020): Tiempo de jugar, tiempo de morir: Apuntes sobre Until Dawn y Man of Medan (Supermassive Games, 2015 y 2019), en Miguel Hernández Communication Journal, Vol. 11 (2), pp. 163 a 180. Universidad Miguel Hernández, UMH (Elche-Alicante). DOI: http://dx.doi.org/10.21134/mhcj. v11i0.335 


\section{Time to play, time to die: Notes on Until Dawn and Man of Medan (Supermassive games, 2015 and 2019)}

Aaron Rodríguez Serrano | serranoa@uji.es

Universitat Jaume I, Castellón (España)

\author{
Abstract \\ Keywords \\ "Death"; "Man of Medan"; “Narratology"; "Until \\ Dawn"; "Video Games". \\ Summary \\ 1. Introduction: The structure and the series. \\ 1.1 Death as the closure of the story. \\ 1.2 Dying in a video game. \\ 2. Suppermassive Games: Evilness and death. \\ 2.1 Theological evil: About the narrators. \\ 2.2 From the evil of men to the evil of the gods. \\ 2.3 How to escape death: cognitive tools. \\ 2.4 How to think about death: symbolic tools. \\ 3. Conclusions. \\ 4. Bibliography.
}

This article aims to make an approach from the textual analysis of the games Until Dawn and Man of Medan (Supermassive Games, 2015 and 2019), based on the problems posed by the concept of "death" in the video game. To do so, both games will be analysed around the following factors: firstly, a brief analysis will be made of what dying in the video game implies in relation to the theories of textual closure - the so-called "classic story mode" and its relationship with the meaning of the texts. Secondly, we will proceed with the analysis of the games themselves, starting from two guidelines: the nature of evil - in its human (essentialist) and supernatural (literary) dimensions - and the position that both games take on death - in terms of mechanics and cognitive resources, but also in terms of its symbolic dimension. Finally, the conclusions are offered and some suggestions are made regarding the future of the research.

\section{How to cite this text:}

Aarón Rodríguez Serrano (2020): Time to play, time to die: Notes on Until Dawn and Man of Medan (Suppermasive games, 2015 and 2019), in Miguel Hernández Communication Journal, Vol. 11 (2), pp. 163 a 180. Universidad Miguel Hernández, UMH (Elche-Alicante). DOI: http://dx.doi.org/10.21134/mhcj. v11i0.335 


\section{Introducción}

En la compleja lectura narratológica de los relatos videolúdicos todavía no se ha pensado con la suficiente profundidad la discusión semiótica tradicional entre la serie y la estructura. La propia definición de la interactividad como un intercambio de posiciones estructurales entre un jugador y un sistema lúdico previo -aunque en ocasiones tan poetizado como en la lectura deleuziana de Cremin (2016)- encaja bien con la propia serialidad del diseño videolúdico -al menos, en aquellos videojuegos, digamos, narrativamente más convencionales.

Dicho con más claridad: más allá del género, de su parámetros narratológicos básicos o de su inevitable carácter abierto, casi todo videojuego puede ser pensado como una suma serial de experiencias lúdicas estructuradas. Ese "casi” que hemos situado en cursiva respondería, como quizá sospeche el lector, a los universos lúdicos abiertos, los videojuegos de carácter más radicalmente “experimental” o, en el límite, ciertas narrativas procedurales (Marcos Molano et al., 2019). Para todo lo demás, la curva de aprendizaje del videojugador se despliega de manera seriada (niveles, fases, habilidades) a partir de una interacción narrativa delimitada (atacar, defender, disparar, hablar) cuyo límite, como es bien sabido, queda trazado por aquello que Victor Navarro definió con gran precisión como la libertad dirigida (2016).

Es sin duda en los trabajos a propósito de las conexiones entre serie y estructura de Eco (1992) donde, con mayor claridad, se puede iniciar lo que podríamos denominar, tentativamente, un "estudio estructural del videojuego". Ahora bien, en el caso que aquí nos ocupa, lo que llamará nuestra atención durante las siguientes páginas es, propiamente, la clausura de la estructura, esto es, la idea misma del cierre del relato que, en una rápida y atropellada comparación con la experiencia humana, apunta directamente a la cuestión de la muerte.

Obviamente, para encarar la cuestión tendremos que valernos de una metodología eminentemente cualitativa, basada en los mecanismos de análisis textual (Marzal Felici \& Gómez Tarín, 2015: 22-25). Para ello, tomaremos un enfoque híbrido que respete los enfoques ontológicos propios del videojuego (Pérez Latorre, 2012; Planells, 2015) con una reflexión que atienda tanto a los rudimentos propios del análisis formal audiovisual convencional (Martín Núñez, 2011) o semiótico (Pérez-Latorre et al., 2017) como a cuestiones derivadas de la narratología y la fenomenología.

Tomaremos como objeto de estudio privilegiados dos videojuegos del estudio Supermassive Games que comparten un gran número de peculiaridades narrativas, formales y mecánicas: Until Dawn (2015) y Man of Medan (2019). Este último título, además, al servir como presentación de una serie de aventuras gráficas cortas englobadas bajo la etiqueta The Dark Pictures tendrá especial interés en lo que a su naturaleza serial. Ambos objetos de estudio pueden justificarse en tanto entre ellos hay una diferencia de cuatro años en los que se aprecia la voluntad clara de un estudio concreto de explorar las relaciones entre mecánicas de juego y muerte, así como una íntima coherencia en lo que toca al diseño de decisiones, pero también a los mimbres del relato en los que se insertan. Si bien se hubiera podido incorporar a la presente investigación otras aventuras gráficas que se relacionan tangencialmente con esta problemática -desde Detroit: Become Human (Quantic Dream, 2018), quizá más cercana a los problemas del posthumanismo, hasta Death Stranding (Kojima Productions, 2019)-, lo que nos ha invitado a reflexionar sobre ambos títulos es la riqueza con la que combinan en su aproximación a la muerte materiales Pulp, citas intertextuales, y una notable autoconsciencia que, pese a su aparente liviandad, permite acometer reflexiones de largo recorrido. En diálogo con otras obras que toman la muerte como 
eje narrativo (Gómez-García et al., 2019), los juegos aquí elegidos se fundamentan en una visión menos realista, pero sin embargo, dejan caer todo el peso de sus implicaciones en el propio devenir videolúdico.

Para ello, seguiremos el siguiente recorrido: en primer lugar nos preguntaremos por la figura narratológica de la muerte como sinónimo de la "clausura" del relato, así como las implicaciones que la muerte tiene dentro de un universo videolúdico. Posteriormente, pasaremos al análisis concreto de los juegos desde el eje de la pregunta por el mal y, posteriormente, desde la pregunta por la muerte misma. En el primer caso nos serviremos del análisis del sistema de narradores para desbrozar las dos grandes naturalezas de la maldad en los mundos de Supermassive Games: el mal esencialmente humano y el mal sobrenatural. En el segundo, pasaremos a ver cómo la idea de la muerte configura los apartados lúdicos y estéticos de ambos juegos. Finalmente, concluiremos el texto con las preceptivas conclusiones.

\title{
1.1 La muerte como clausura del relato
}

En un reciente y muy estimulante trabajo de Roberto Amaba (2019), el teórico plantea un esfuerzo notable por hibridar los lugares comunes de la narratología audiovisual con las llamadas "atracciones" o elementos de la experiencia vinculables a la pura descripción, quizá mostración que configuran un cierto universo diegético. Entre los muchos retos que allí se encuentran desarrollados, uno especialmente brillante tiene que ver con los motivos por los cuales la todopoderosa "clausura del relato" - citada de manera habitual como una de las garantías propias del "modo del relato clásico" en oposición a un hipotético "modo del relato postclásico" (González Requena, 2006)- debería ser puesta en duda en un nivel casi esencialista, conceptual:

\begin{abstract}
Así las cosas, los juegos con la clausura del relato afectan a la sutura del espectador. Películas que juegan con la baraja marcada en busca de tres objetivos. Primero, disfrazar la participación del espectador de interactividad. Segundo, sugerir un respeto no siempre sincero hacia sus capacidades cognitivas. Tercero, habilitar una serialidad amparada en la demanda fisiológica de ese espectador seducido pero frustrado en el interruptus. Pura estrategia de guión, digo de mercado. La prolongación o está planificada o viene abalada por el libro de cuentas (Amaba, 2019:134).
\end{abstract}

En efecto, la hipotética "ausencia de clausura" ni garantiza una mayor participación del receptor del texto, ni es garantía de mayor exigencia estética o de mayor seriedad en su discurso, ni muchísimo menos escapa de lo que no deja de ser, en términos casi fenomenológicos, una constante y agónica exigencia inconsciente de sentido. Al hablar de sentido tomamos como referencia precisamente a dos autores de la escuela francesa que serán de gran utilidad en las siguientes páginas: por un lado, la distinción inicial entre las significaciones del término esbozadas desde una perspectiva hermenéutica por Gadamer (Gadamer, 1977) y, posteriormente, la ampliación desarrollada por Jean Grondin en su obra monográfica sobre el tema (2018). Grondin, concretamente, señala cuatro acepciones del término que están inevitablemente conectadas con la búsqueda que aquí iniciamos:

a) El sentido direccional: Una huella, una línea de fuerza que se traza en el videojuego a partir de los movimientos del personaje, pero también de los objetos que configuran el mundo (las balas disparadas, la dirección de los pasillos).

b) El sentido significante: El intercambio social entre personajes que en ambos juegos se anuda en torno al lenguaje -así como la modificación de sus conductas, de sus psicologías, de sus gestos mismos- a partir de las posibles interpretaciones que el interfaz del juego propone sobre su mostración. 
c) El sentido sensitivo: Un sentido que cae exclusivamente del lado del videojugador o videojugadora, y que sería la inevitable aprehensión que se produce al perder a un personaje por una mala decisión -acto irrevocable, como veremos, en ambos juegos-, y que modifica el valor concreto de las vidas de los mismos así como la transmisión de la responsabilidad en la toma de decisiones que los juegos establecen.

d) El sentido reflexivo: Que es, obviamente, el que surge a posteriori de la experiencia de juego y propone un replanteamiento de las propias coordenadas metafísicas que rodean a cada jugador concreto.

Como vemos, los dos primeros niveles quedan de alguna manera anudados en la complejidad de la cuestión del sentido que viene dada en ese juego doble que ya esbozó Deleuze: "El sentido es lo expresable o lo expresado en la proposición, y el atributo de estado de cosas" (Deleuze, 1989: 50), mientras que los dos segundos quedarían de alguna manera superados, imposibles de clausurar, quizá más cercanos a esa superación que Adorno ya leyó (2006: 89-95) en algunas de las intuiciones de Kierkegaard.

Nos guste más o menos, toda clausura material, real del relato -ya sea el final de la película, el momento en el que se apaga la consola o se cierran las páginas de un libro- agota todas las posibilidades estrictas de interpretación, y por lo tanto, limita el proceso hermenéutico. El propio González Requena señala su conexión con la cuestión del sentido cuando afirma: "el fin, el cierre, la clausura o la muerte, como se prefiera, cristalizan los actos y los dotan de sentido" (González Requena, 1988: 119). No hay sentido más allá del texto: hay comentarios, interpretaciones, hipótesis, posibilidades de ciertos decires que en el mejor de los casos partirán de los materiales textuales y, en el peor, se desplomarán directamente en la pura ciencia ficción analítica o en los flujos más salvajes del fan fiction (Hidalgo Marí \& Sánchez Olmos, 2015). Pero el sentido, en un nivel realmente auténtico y radical, únicamente queda depositado en los procesos de significación que tienen lugar y se despliegan a partir de los operadores textuales, es decir, de lo que ocurre dentro del texto - la "toma de postura en la que yace su compromiso íntimo con la verdad”, como apuntaron Martín-Núñez y García-Catalán (2015: 349). Por poner un ejemplo concreto: por mucho que el jugador o la jugadora desee encontrar una lógica a esos pilotos anónimos que recorren interminablemente los circuitos de WipEout Omega (Sony, 2017), no encontrará rasgo alguno desde el que le permita acceder a ese proceso lógico. A la inversa, un improbable jugador o jugadora de Star Wars Jedi: Fallen Order (Respawn Entertainment, 2019) que decidiera comenzar una partida sin haber transitado jamás uno de los incontables textos del universo Star Wars, entendería el "significante-Fuerza" como lo que, en el fondo, es: una pura mecánica ludonarrativa que permite modificar los parámetros tiempo, densidad o espacio, sin que ningún rasgo narrativo -vinculado o no a los midiclorianos- le acompañe.

Este desvío es necesario porque la muerte, en nuestra pequeña gran experiencia cotidiana del mundo, provoca un efecto paralelo. Esa era, quizá, la llave maestra que abría la complejidad de la ontología existencial de Martin Heidegger (2009), y que de manera inevitable, sobrevolará en las siguientes páginas. La muerte (propia) funciona como un corte definitivo con los procesos de significación que, mejor o peor, uno ha tenido la posibilidad de sugerir con su propia eksistencia. Ciertamente, no hay idea tan materialista, y a la vez, tan inevitable: únicamente a partir de mi propia muerte se puede trazar un sentido por los momentos recorridos, las decisiones tomadas, las acciones ejecutadas y, por supuesto, el delgado hilo de deseos que han conducido de cada una a la otra. Estamos, como el lector sospechará, acariciando los mapas de decisiones 
propios de los juegos de Supermassive Games. Pero todavía debemos demorarnos un poco más.

\subsection{Morir en un videojuego}

La muerte en un videojuego siempre tiene algo de paradójico. Quizá porque, en el fondo, la muerte en los universos videolúdicos no suele ser sino una dinámica.

Ciertamente, este argumento mismo exige una mirada cuidadosa en tanto acabamos de formular una imposible contradictio in terminis. De lo muerto se presupone, en principio, que no tenga especial capacidad de acción o movimiento. Por supuesto, lo muerto genera efectos en los que sobreviven -rasgo que, paradójicamente, no suele ser tenido en cuenta en los juegos de Supermassive Games-, pero se suele tratar de modificaciones simbólicas, replanteamientos sobre las fantasías del lugar que ocupamos en el mundo, ejercicios más o menos melancólicos. No podemos detenernos aquí en la complejidad de la avataridad-cuya síntesis, por otro lado, se encuentra impecablemente desplegada en un texto reciente de Navarro Remesal (2019)-, si bien tendremos sus implicaciones como fondo de las siguientes páginas.

En casi todos los videojuegos la muerte aparece como un gesto inevitable dentro de la aventura que queda más o menos vinculado con la propia curva de aprendizaje del resto de interacciones. Para aprender hay que morir -lo que sin duda, no deja de ser un enunciado simbólico-, aunque dicho estado no deje de ser una molesta y transitoria actividad limitante de nuestras potencias dentro del juego -en World of Warcraft (Blizzard, 2005) o en No man's sky (Hello Games, 2016), por ejemplo.

A poco que lo piensen, verán como hay pocos juegos en los que la idea de muerte -cualquier idea de muerte real, por pequeña que sea- no esté escrita de manera más o menos explícita en su tejido narrativo. Tomemos un caso peculiar: Pokemon Let's Go Pikachu! (Nintendo, 2018) es un juego en el que, a primera vista, resulta prácticamente imposible morir: el protagonista puede ser derrotado y sus criaturas vencidas, pero la amenaza de su desaparición explícita en el mundo diegético no se contempla como una posibilidad misma del tejido narrativo. Era una mecánica que no se contemplaba tampoco en su anterior edición de realidad aumentada - cuya limitación en las mecánicas ya ha quedado estudiada en trabajos anteriores (A. Rodríguez Serrano et al., 2017). En el caso más reciente de la reedición para Nintendo Switch, a poco que el jugador decida invertir el tiempo suficiente en coleccionar, entrenar y enfrentar a sus criaturas resulta poco menos que imposible que agote la línea principal del relato.

Ahora bien, precisamente en ese tránsito por el mundo narrativo resulta imposible no toparse con Pueblo Lavanda, escenario específicamente construido a partir de los mecanismos de duelo y recuerdo. Más allá del sonrojante tono amarillista con el que Pueblo Lavanda fue tratado por los medios divulgativos españoles, lo cierto es que funciona como un interesante tejido simbólico en el que, pese a la imposibilidad de morir por parte del protagonista, Satoshi Tajiri y su equipo incorporan la cuestión misma del proceso de la muerte de una manera tangencial: entrenadores que recuerdan a sus criaturas perdidas, fantasmas que no han cumplido con sus ritos funerarios, espacios rituales para los enterramientos, las oraciones y las ceremonias... En muchos aspectos, la saga Pokémon se posiciona como una suerte de coming of age, de narración sobre los ritos iniciáticos que separan la infancia de la adolescencia, simbolizando los habituales umbrales campbellianos (2005) mediante una progresión mediada de gimnasios, entrenadores y encuentros con los diferentes villanos del Team Rocket. Por mucho que las aristas queden razonablemente disimuladas bajo el aparataje gráfico, la suavidad de los elementos que configuran la experiencia 
estética e incluso la amabilidad de las mecánicas de combate, la conexión entre muerte y rito de paso está literalmente escrita en la superficie del juego.

\section{Supermassive Games: El mal y la muerte}

La relación de estos dos videojuegos con el Slasher parte de una muy difusa relación con la muerte que anuda al siempre inevitable conjunto de jóvenes norteamericanos atractivos, de clase alta, con estudios superiores, despreocupados e ingenuos con una serie de amenazas inevitables que se sitúan en su campo de acción inmediato. La sensación de aislamiento - un refugio nevado en Until Dawn, el mar abierto en Man of Medan- se estructura en torno a dos tipos de amenazas que cierran el círculo del mal: asesinos humanos de aspecto amenazador -Josh (Rami Malek) y su particular performance demencial en el primer caso, la banda de secuestradores en el segundo- o bien criaturas extraídas del folclore sobrenatural -los wendigos y los cadáveres de la tripulación del barco en la segunda. Si comparamos su diseño estructural básico en términos narrativos (Petridis, 2019) -incluyendo las inevitables ironías autocumplidas sobre la muerte (Ménard et al., 2019)- vemos que los mecanismos de intertextualidad siguen un patrón claramente postclásico, propio de los estilemas de nuestro tiempo (Palao Errando et al., 2018). Ahora bien, la profundización en aquellos gestos específicamente vinculados con la existencia del mundo son bastante más complejos y requerirán, por tanto, un trabajo algo más refinado.

\subsection{El mal teológico: Sobre los narradores.}

Es interesante que la cuestión de la causa del mal sea ontológicamente difusa en ambos universos. Que el mal existe es algo que el videojugador deberá experimentar prácticamente desde ambos prólogos: el problema, por supuesto, es saber por qué. De hecho, hay algo implacable a propósito de su inevitabilidad que tiene que ver directamente con las mecánicas: en ambos openings queda claro que no importa lo diestro que sea el jugador o lo sagaz que sea en su acción detectivesca o su toma de decisiones: la muerte debe acontecer porque así está escrito en el programa narrativo del juego y, guste o no, pasa por encima de la propia libertad de acción o las habilidades del jugador. Hay una primera causa del mal, por lo tanto, que es inherente a la narrativa y a las propias leyes causales del universo. Son mundos heridos de algo así como una especie de inflexibilidad teológica, atravesados por la caprichosa sonrisa de un narratorzuelo cruel que se complace en desprenderse despreocupadamente de sus criaturas.

Esta "causa del mal" resulta interesante, además, en tanto tiene una suerte de trasunto narrativo incorporado en la propia historia: esos demiurgos sarcásticos que, desde el exterior de la trama principal, van comentado el relato, ofreciendo opciones o pistas al videojugador, pero ante todo, imponiendo una cierta distancia con respecto a los personajes principales.

${ }^{1}$ Es interesante señalar que, pese a esto, Until Dawn todavía ofrecía una pequeña posibilidad en su segunda mitad al descubrir que había una posibilidad incluso peor que la muerte misma que se había jugado en el prólogo. Cuando se descubren los objetos personales de Hannah y Beth, el diario arroja que la primera no murió en la caída sino que fue condenada a una lenta agonía e incluso a comerse el cadáver de su hermana para sobrevivir. En uno de los árboles de decisiones es posible incluso volver a encontrarse con la propia Hannah, convertida ahora en un wendigo y demostrando ese viejo motivo del género del terror: que la imaginación humana puede incluso fantasear destinos peores que la muerte misma. 
Detengámonos brevemente en ellos. Por un lado, en Until Dawn contamos con una suerte de psicólogo que puntúa los diferentes capítulos y que pertenece a un mundo que se va descomponiendo progresivamente. Por otro lado, en Man of Medan el demiurgo escribe y narra la historia a la vez, remitiéndose a ese doble juego de interacción/estatismo propio de las películas interactivas.

La relación de ambos personajes con el mal es, de nuevo, difusa, y a nuestro juicio resulta inseparable a la construcción estética y lúdica que juegan en el interior mismo del relato. Ambas figuras, masculinas, están situados directamente en espacios vinculados con el saber y con la palabra. Dominan los flujos afectivos que enhebran la trama con una suerte de presencia de clarísimas resonancias teológicas. Veamos ambos casos.

En Until Dawn, la voz de la ley tiene un sabor inevitablemente terapéutico. La "consulta" funciona como una especie de territorio biopolítico: el cuerpo del jugador está bloqueado -no podemos movernos por su interior- mientras que las mecánicas de interacción se resumen más bien a mirar, señalar entre pares de objetos o alternativas... A nivel formal, esto privilegia la supremacía del demiurgo: retratado generalmente de manera frontal, en plano medio (Fig. 01) o en primer plano, su encarnación del poder y de la ley no deja lugar a dudas: el suyo es un rol de mandato sobre nosotros -si bien, por supuesto, no puede ser pensado en términos de transferencia psicoanalítica, sino al contrario como una especie de encarnación del super-yo en un sentido netamente obsceno (Lacan, 1981).

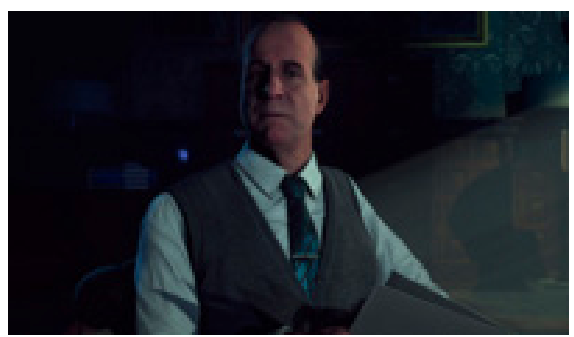

Fig. 01: Until Dawn (Supermassive Games, 2015). Supermassive Games @

En oposición, tenemos al narrador/relator de Man of medan, cuya dimensión es en principio bastante más amable y cuyos antecedentes intertextuales apuntan en una dirección bien distinta. El carácter serial de la obra - del que hablaremos más adelante- parece conectar su figura con aquellas sarcásticas intervenciones acuñadas por Alfred Hitchcock en su serie Alfred Hithcock presents... (Castro de Paz, 1997), y que después fueron apropiadas y desarrolladas en diferentes referentes tanto televisivos - Tales from the crypt (HBO, 1989-1996)- como cinematográficos -Creepshow (George A. Romero, 1982).

Al contrario que en Until Dawn, su función no es tanto intervenir en la historia como comentarla, guiar una cierta lectura, e incluso en el límite, mostrar una inquietante empatía frente al aciago destino de sus protagonistas. El espacio virtual ha sido desplazado del campo del cuerpo y la terapia (una consulta) al campo del saber literario (una biblioteca de resonancias góticas), aunque la capacidad de interacción del videojugador ha sido reducida hacia su más mínimo exponente.

Sin embargo, no hay que equivocarse: aunque su rostro sea más amable, el narrador de Man of Medan sigue siendo una figura eminentemente teológica, e incluso de una dimensión más trágica todavía si cabe. Al observar su propia construcción estética en plano (Fig. 02) resulta inevitable 
darse cuenta de la cercanía de ambas figuras: misma escalaridad, mismo vestuario (chaleco, corbata, camisa impecable).

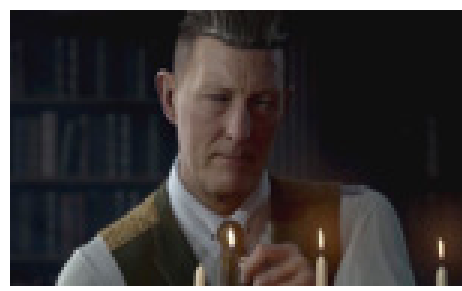

Fig. 02: Man of Medan (Supermassive Games, 2019). Supermassive Games (C)

La diferencia de tono no esconde el recorrido al que apuntan ambos personajes: a la explicitación teológica de un mal que gobierna el universo lúdico y del que emergerán los diferentes conflictos que dominarán las curvas de transformación de los personajes. Las primeras intervenciones del narrador de Man of Medan están, además, acompañadas de la Lacrimosa del Réquiem de Mozart como fondo musical, subrayando lo ominoso de la estancia pero, ante todo, la conexión con lo que se está jugando en ese espacio: la muerte.

Es necesario tener en cuenta que las palabras del personaje se superponen con un texto original religioso muy concreto que dice lo siguiente:

Lacrimosa dies illa/Qua resurget ex favilla/Judicandus homo reus./Huic ergo parce, Deus:/Pie Jesu Domine,/Dona eis requiem. Amen. [Ese día en el que lloraremos/en el que resurgirá de la ceniza/El hombre culpable para su juicio/Piadoso Señor Jesús/Otorga el descanso eterno, Amén - Traducción propia]

Lo que está en juego, por lo tanto, es la cuestión del juicio tras la muerte, que como es bien sabido, es un mecanismo eminentemente religioso para dar sentido tanto a la propia muerte (debemos atravesar ese umbral para acceder a esa Tierra Prometida) como al hecho mismo de la ordenación ética de los actos de nuestra vida (hay un Dios que juzga). Y ya puestos, no hay que olvidar que la idea misma de Juicio Final apunta a la idea de que hay una consecuencia sobre las acciones que tomemos, base central sobre la que se asientan los últimos juegos de Supermassive Games.

Luego, llegados a este punto, quizá no resultaría demasiado descabellado concluir que tanto el terapeuta como el narrador son las dos caras de la misma moneda: una figura paterna que castiga y aterroriza, y al tiempo, una figura paterna que comprende y empatiza con el sufrimiento de sus hijos. Es el territorio de ese "sueño infantil en el que, de forma prototípica, la imagen del padre está desdoblada entre el padre amable y el padre monstruoso" (Parrondo, 2014: 163)

Padre simbólico total, en cualquier caso, y por lo tanto, vinculado con la problemática misma de la existencia del mal. Padre, en esencia, que goza además de esa misma existencia: ya sea por la vía de la "hipotética terapia" - la ausencia o la parquedad de las mecánicas de interacción nos aplasta, nos recuerda constantemente nuestra pequeñez, nuestra impotencia ante su figura- o por la vía del "amor impostado" - ese supuesto alivio que atraviesa al narrador cuando conseguimos salvar a los personajes.

\subsection{Del mal de los hombres al mal de los dioses}

Como señalábamos hace unas páginas, una vez se desciende hacia el corazón del relato, la presencia explícita del mal adquiere siempre un doblez, una ambigüedad, en la que no siempre 
resulta fácil distinguir hasta qué punto lo que transitamos no es sino una consecuencia directa de actos estrictamente humanos - mascaradas, bufonadas salvajes, extrañas políticas bélicas de exterminio-, o elementos propios del género sobrenatural. Esa extraña mixtura que va configurando las formas en las que la muerte se manifiesta hace que la toma de decisiones llegue incluso a enrarecerse. Tomemos un par de ejemplos concretos de Until Dawn.

La imaginación de Josh obliga al videojugador a elegir quién debe morir en dos aparentes callejones sin salida: la supuesta encerrona con un mecanismo de despiece que siempre se salda con la (falsa) muerte del anfitrión, pero también la mucho más compleja decisión con la pistola trucada que obliga a elegir a Chris si decide dispararse a sí mismo o, por el contrario, sacrifica a Ashley (la mujer que ama) para su propia supervivencia. En ambos casos la elección del videojugador resultará inútil: haga lo que haga, ningún personaje morirá. Lo que impregna el relato, por supuesto, es la desazón misma por haber tenido que elegir, la certeza misma de que cierto problema ético exige urgentemente -el tiempo es clave- una muy personal solución.

Estos dos ejemplos se vinculan a un mal esencialmente humano. La acepción de lo esencial debe leerse aquí en toda su literalidad: se vincula con nuestra supervivencia como especie, con los mecanismos cognitivos que nos llevan a elaborar una serie de cálculos incontrolables con respecto a nuestros intereses y a las consecuencias de nuestras acciones. Del mismo modo, la responsabilidad es también una variable inevitablemente humana: los actos se hubieran podido evitar, el gesto del videojugador hubiera podido optar (quizá así lo hizo) por situarse en esa compleja zona gris de responsabilidad ética en la que no tomar ninguna acción es, consecuentemente, tomar una acción.

Es necesario señalar, además, que esto no ocurre así en Man of Medan. Una diferencia sustancial en el despliegue de la trama es la manera en la que el juego, queramos o no, nos obliga a no permanecer en esa zona gris ética -o, por el contrario, nos castiga si así decidimos hacerlo. Valga como ejemplo el momento en el que uno de los piratas, enloquecido, amenaza con disparar a dos de los personajes si no se le convence de lo contrario. El videojugador puede optar por guardar silencio, en cuyo caso inevitablemente Chris será disparado en la cabeza. Volveremos, no obstante, en algunos epígrafes al problema de la esencialidad de este tipo de mal frente a la muerte $^{2}$.

\footnotetext{
${ }^{2}$ Una consideración aparte debería hacerse, aunque sea con forma tangencial de excurso, a propósito de Intenciones ocultas (Hidden Agenda, Supermassive Games, 2017), un juego que funciona como "bisagra" en muchos aspectos entre Until Dawn y Man of Medan. En aquella ocasión, la problemática del mal adquiere tonalidades mucho más radicales, ya que es posible terminar de manera definitiva la partida si se toman ciertas acciones éticas concretas. Por poner un único ejemplo, en un momento de la narración nos vemos ante la alternativa de escapar de una casa en la que se encuentra un personaje secundario atrapado con una bomba injertada, o por el contrario, acompañar al sufriente en sus últimos momentos. Si el videojugador decide permanecer en la casa e intentar hasta el último segundo el acto de salvación, la bomba explota y la partida termina: es necesario recomenzar la aventura desde el principio. Esta quiebra tan brusca del árbol de decisiones no se encuentra en los otros dos títulos que aquí nos ocupan y configura, todo hay que decirlo, una de las decisiones de ética ludonarrativa más interesantes de los últimos años.
} 
El mal se manifiesta en los actos humanos a través de una serie de referencias, conexiones y redes simbólicas expansivas poco menos que vertiginosa: hay un mal biopolítico (los cuerpos enfermos, la ausencia de empatía, la sociopatía y los mecanismos de control), un mal sistémico vinculado al despliegue del capital -la mina que se hunde en Until Dawn, los países en vías de desarrollo de los que parecen emerger los piratas de Man of Medan-, un mal histórico que forma parte de nuestra herencia como sujetos - piénsese en las constantes referencias a la II Guerra Mundial de Man of Medan, que se incorpora así al apasionante debate abierto sobre la ficcionalización y la pertinencia de usar dicha contienda con intereses ludoficcionales (Rodríguez Serrano, 2014). Hay una intimidad que emerge de la complejidad afectiva de los personajes en muchas ocasiones podemos decidir la manera en la que se humillan, enfrentan, denigran entre ellos-, pero que se va expandiendo progresivamente a través de las historias familiares, las estructuras económicas, los escenarios geopolíticos hasta llegar, finalmente, a su manifestación no-bumana.

Ahí es precisamente donde podemos apreciar cómo esa capa esencialista emerge, se superpone y se fusiona, a su vez, con una capa final que, de manera provisional, podríamos denominar sobrenatural. Los Wendigos de Until Dawn, los monstruos primigenios y las visiones de Man of Medan se mueven entre la alucinación personal y la evidencia explícita de su existencia. Emergen del humus mismo del fantástico literario y se reactualizan en torno a una serie de parámetros que ambos universos suelen dejar en suspenso, quizá recreándose más en la citación, el guiño cómplice, el placer del reconocimiento. Así, por ejemplo, Man of Medan incorpora invocaciones y sectas que apuntan a la construcción textual de Lovecraft, pero también jump scenes propias del cine de terror contemporáneo. En el mismo catálogo juegan escenas tan pueriles y encantadoras como el "relato de terror" que improvisa Chris para sus compañeros de viaje al comienzo de la tormenta, que parece íntimamente conectado con las historias de campamento, pero también con narraciones adolescentes seriales como Are you afraid of the dark? (YTV, 1990) -que, todo sea dicho, contó con una meritoria y hoy prácticamente olvidada adaptación al videojuego.

La fusión de ambas capas acaba disparando inevitablemente esa suerte de ambigüedad narrativa en la que el mal -y por extensión, la muerte como su manifestación definitiva- no queda teleológicamente definido, sino que simplemente, es.

\subsection{De cómo escapar a la muerte: Herramientas cognitivas.}

Una vez desbrozado -o al menos, sintetizado- el problema de las causas del mal, ya resulta posible acercarse a la cuestión concreta de la muerte.

En primer lugar, ambos videojuegos se construyen en torno a un único objetivo principal: evitar que muera el mayor número de personajes. Al contrario de lo que ocurre en el slasher cinematográfico -que parece hundir sus mecanismos espectaculares en la contemplación explícita de las muertes seriadas de sus protagonistas-, lo que motiva al videojugador es escapar precisamente de aquellas situaciones narrativas en las que alguien muere. Llegar al final con el mayor número de supervivientes implica también -en términos de focalización- acceder a la mayor cantidad posible de información sobre el universo ludonarrativo. Es cierto que las líneas básicas del relato suelen pasar por el personaje principal. En Until Dawn el caso evidente es el de Samantha que, por lo demás, sigue el patrón argumental tradicional de la Final Girl del género. Sin embargo, mantener a los personajes activos y razonablemente cohesionados permite un mayor acceso a los materiales narrativos. 
En esta dirección, el primer mecanismo jugable que debería llamarnos la atención es la existencia de ítems que permiten acceder al posible futuro de los protagonistas -los tótems o los cuadros. Curiosamente, y en discusión con lo que sugieren ciertas interpretaciones metafísicas sobre dicho elemento (Siabra Fraile, 2012), esta mecánica no modifica sustancialmente los aspectos internos del universo -los personajes no ganan atributos, no se "desbloquea" acceso espacial o jugable alguno-, sino que simplemente se ofrece un posible desarrollo temporal. En ocasiones, el videojugador no podrá interpretarlo, en otras ni siquiera tendrá relación con el árbol de decisiones que se recorre en la partida.

Saber puede tener que ver con la muerte, pero no es necesariamente ninguna garantía. Además, ese "saber" tiene una dimensión compleja que pasa explícitamente -lo que no deja de tener cierta ironía- con el propio análisis textual narratológico. En términos de relato, lo que un tótem o un cuadro ofrecen es un fragmento de duración externa al desarrollo de la partida, una dislocación sobre el presente que exige una interpretación en términos de montaje -¿qué ha podido llevarnos a esa situación?- como de puesta en escena-¿qué es lo que esconde la cámara desde esta angulación concreta?

Poder descifrar exige al videojugador que, antes que nada, se familiarice con los mecanismos estrictamente visuales de ambos juegos. Si tomamos como referencia la segunda parte del prólogo de Man of Medan pronto veremos cómo el manejo de cámara parece falsear constantemente una mirada propia del cine de terror. Los pasillos del barco se alargan mientras la cámara asciende o desciende verticalmente en relación con el cuerpo del avatar. Los sustos se suelen reservar para los planos subjetivos en los que abrimos una taquilla o inspeccionamos un objeto. Las pruebas de habilidad en las que el personaje debe esconderse o actuar con celeridad suelen retratarse en planos americanos o medios, casi siempre en composiciones descentradas que dejan grandes áreas a izquierda o derecha.

En todos los casos, resulta factible formular -aunque sea en una dimensión estrictamente metafórica- que es la muerte misma la que está mirando. La cámara se experimenta como una amenaza, como escritura de la fragilidad, el pánico ante la situación. El movimiento de los personajes queda además limitado por pasillos angostos y de cierta profundidad, lo que restringe notablemente la información visual concreta del espacio y, por extensión, condena a las líneas de movimiento a ser poco menos que trayectos morosos, interminables.

Este aprendizaje de los usos de la cámara - en el que se podría incorporar también el montaje picado a gran velocidad de las secuencias de acción o la manera en la que los primerísimos primeros planos sirven para alertar también de amenazas inmediatas- resulta fundamental para realizar una correcta lectura de los acontecimientos futuros. Otro tanto puede decirse de los elementos que configuran el profílmico del videojuego: los objetos con los que se cometen los crímenes, las estancias en las que tienen lugar y, por supuesto, qué caracteres concretos las sufren. Una llave inglesa, un cuchillo, una puerta con una cierta inscripción o una escalera levemente iluminada sugieren una cierta anticipación que mejora, aunque sea de manera aproximada, las posibilidades de sobrevivir - otras defunciones, como las de Chris en Until Dawn o la de Brad en Man of Medan dependen simplemente de la capacidad de reacción del videojugador para enfrentarse con una correcta puntería o una certera combinación de botones a un único reto puntual.

Aprender a mirar, re-conocer, trabajar desde la lógica de la enunciación (gestión de la mirada y sobre el profílmico) son actividades estrictamente intelectivas y dependen de procesos lógicos de procesamiento de la información por parte del usuario. Acertar en la combinación o apuntar son actividades opuestamente físicas, de agilidad cognitiva y basadas en la pura rapidez fisiológica 
ante una cierta amenaza. Las dos están vinculadas, por lo demás, a los propios mecanismos de desarrollo de nuestro cerebro, se hunden en la noche de los tiempos y se encuentran en la naturaleza misma del juego como aprendizaje: Supermassive Games apela, así, a nuestro pasado remoto como especie ante la presencia misma de la muerte.

\subsection{De cómo pensar la muerte: Herramientas simbólicas.}

No queríamos dar por concluido el texto sin apuntalar, brevemente, el problema principal que convocan ambos juegos: la inquietante irrupción de una forma indudablemente más compleja de pensar -simbólicamente- la naturaleza de la muerte dentro del universo videolúdico.

Como señalábamos hace unas páginas, el funcionamiento mismo de la repetición como gesto común en los juegos (Loriguillo-López \& Sorolla-Romero, 2015), ha parecido sugerir una cierta sublimación de la idea misma de muerte. Pensemos, por ejemplo, en el ángel naïf que ascendía a los cielos tras cada derrota en Alex Kidd in Miracle World (Sega, 1986). Morir no era sino la promesa de una resurrección inmediata, o como poco, la penalización inevitable en el transcurso de una serie de adquisición de saberes y habilidades por parte del videojugador.

Lo que realmente nos ha parecido meritorio de Until Dawn y Man of Medan es la honestidad con la que el gesto de morir alcanza una dimensión específicamente irrevocable -al menos, en la extensión de una única aventura- y, posteriormente, cómo escribe la manera en la que el mundo, dramáticamente, continúa.

En muchos aspectos, Until Dawn está más cerca de la narración sobre el luto que sobre el slasher mismo. Tras el prólogo, la historia se vincula con la necesidad de restaurar una comunidad rota por la pérdida, de encontrar una cierta forma de canalizar la desesperación de un conjunto de individuos. El hecho de que sea precisamente Josh -el hermano de una de las víctimas- el que pone en marcha la tragedia con sus bromas enloquecidas no hace sino poner frente a nosotros la extraordinaria complejidad de comportarse normativamente allí donde el agujero de la pérdida amenaza con destruir la propia subjetividad.

Man of Medan, a su vez, retoma esta idea, pero con una clara dimensión ideológica. El duelo ya no es una cuestión personal que circula entre una serie de caracteres unidos por un vínculo de amistad. Al contrario, lo reprimido toma una forma militar, se vincula con una serie de políticas injustas, decisiones equivocadas, es una cuestión - por usar un eufemismo de moda para no hablar de los costes humanos ni de los recortes de libertades personales- de "seguridad nacional". Desde el primer minuto se ponen encima de la mesa las diferencias sociales de los personajes principales -sus deudas económicas, las diferencias educativas, la soberbia de sus acciones- hasta el punto de que el propio saqueo de la Historia se convierte en un pasatiempo, una domesticación del mal, una suerte de turismo imbécil para ocupar el tiempo de las clases altas.

Man of Medan habla, en primer lugar, de cómo se reprime el verdadero núcleo de horror del capital. No olvidemos que los protagonistas pagan por visitar espacios en los que se escribe la tragedia histórica y se fotografían junto a los fuselajes de los aviones derribados en combate de una manera muy similar a las inefables colecciones de influencers varios que posan junto a los memoriales del Holocausto o en los escenarios de la masacre de turno. Ahora bien, lo propio de la muerte (de los otros) es, precisamente, su naturaleza como agujero definitivo en el tejido simbólico de la realidad. No es de extrañar que el juego presente el retorno de los muertos y de su imaginería como una fantasía, un delirio, una especie de alucinación. En efecto, la lógica del mecanismo psicótico trae lo 
reprimido a lo real (Lacan, 1984), y con ello, despliega con una claridad demoledora aquello que se había intentado negar sistemáticamente -a golpe de selfie, por ejemplo.

Esta misma idea puede aplicarse, a nuestro juicio, a la manera generalista en la que el videojuego ha tratado la muerte. Al igual que en Man of Medan, para el videojugador convencional la muerte no es sino la parte integrada de una industria del ocio, un entretenimiento, casi un automatismo. En tanto pertenece al campo de la ficción, no deja de ser una especie de operador textual. Ahora bien, precisamente cuando propuestas como las de Supermassive Games irrumpen en el panorama -a las que se podían sumar sin pestañear otros juegos como What Remains of Edith Finch? (Giant Sparrow, 2017) o incluso The Stanley Parable (Galactic Café, 2013)- cabe pensar que algo ha desbordado ese gesto inicial, que lo ha llevado al exceso, o en el límite, que ha conseguido tomárselo en serio.

Como apuntábamos anteriormente, si algo se le puede reprochar a estos juegos en el contexto que nos ocupa es, precisamente, que no lleven su decisión hasta sus últimas consecuencias. Su cierre narrativo concluye generalmente con - de nuevo- la clausura del relato convencional: la huida, la supervivencia, la salvación. La muerte se ha escrito únicamente en tanto pérdida-ya no podemos controlar a uno u otro de los caracteres- pero no se nos ha dado la oportunidad de experimentar, con todo su peso, la abrasadora onda temporal de sus efectos. Ni siquiera Josh, el gran doliente de Until Dawn, sirve para encarnar videolúdicamente lo que ocurre (aquí, entre nosotros) después de la muerte.

\section{Conclusiones}

Con lo que, finalmente, podemos sintetizar mediante algunos breves enunciados cuáles han sido los pasos principales que cierran nuestro trabajo.

Conclusión 01: En primer lugar, hemos realizado una pequeña aproximación al concepto de muerte en el videojuego que, esperamos, pueda sumar alguna sugerencia de valor a los estudios sobre la problemática de la avataridad. A nuestro juicio, lo más interesante es poner en duda la relación entre clausura del modo de relato "clásico" -tal y cómo suele ser estudiado, al menos, desde la teoría del texto y los referentes ya citados- y aquello que entraña sentido -en las cuatro acepciones de Grondin. Si bien hay una correspondencia inevitable entre el concepto de la eksistencia de la ontología existencial y esa misma idea de la muerte como clausura narratológica, en los videojuegos no puede seguirse dicha conexión. Es necesario, por lo tanto, reubicar dichos conceptos en un futuro.

Conclusión 02: Entrando en el análisis textual de los juegos propuestos, nuestro recorrido ha puesto de relieve las siguientes ideas.

Conclusión 02-A: En lo que toca a la naturaleza del mal, hemos visto cómo Supermassive Games se vale de un sistema híbrido que no es reducible a ninguna teleología por la que la naturaleza del mal en el mundo funciona siempre como un rostro bicéfalo: una parte que debería ser pensada desde la ética -el mal en tanto categoría esencial de lo humano- y una parte que deviene formulación mitológica, traslación, reescritura en término sobrenatural. Así, es fácil ver que dicha idea encaja con las teorías propuestas por Campbell (2019) que toman el mundo de los mitos como una suerte de traslación activa y viva de aquellas pulsiones o paisajes interiores que funcionan en la experiencia individual. Algo parecido proponen ambos videojuegos: tras la tramoya de seres mitológicos, bestiales o demoníacos, lo que realmente emerge es una reflexión de gran calado sobre el duelo, la locura o la soledad. 
Conclusión 02-B: La muerte en ambos juegos tiene, por lo tanto, una doble naturaleza que se conecta con lo ya señalado: hay un primer nivel en el que se conjura o se esquiva de manera estrictamente cognitiva (procedimientos lógicos de deducción, pero también reflejos y acciones marcadas por un nivel poco menos que instintual), y un segundo nivel experiencial en el que apunta a la función mítica: procesos de simbolización, de donación de sentido y escritura.

\section{Bibliografía}

Adorno, T. W. (2006). Kierkegaard: Construcción de lo estético. Akal.

Amaba, R. (2019). Narración y materia: Supervivencias de la imágen cinematográfica. Shangrila.

Campbell, J. (2005). El Héroe de las mil caras : psicoanálisis del mito. Fondo de Cultura Económica de España.

Campbell, J. (2019). Tú eres eso: Las metáforas religiosas y su interpretación. Atalanta.

Castro de Paz, J. (1997). Estrategias discursivas en el Telefilm de los años 50: Alfred Hitchcock Presents y la construcción del espectador televisivo. Archivos de La Filmoteca: Revista de Estudios Históricos Sobre La Imagen, 27, 196.

Cremin, C. (2016). Exploring Videogames with Deleuze and Guattari: Towards an affective theory of form. Routledge.

Deleuze, G. (1989). Lógica del sentido. Paidós.

Eco, U. (1992). Obra abierta. Planeta-Agostini.

Gadamer, H. G. (1977). Verdady método : fundamentos de una hermenéutica filosófica. Sígueme.

Gómez-García, S., Navarro-Sierra, N., \& Carrillo-Vera, J.-A. (2019). De la lucha al testimonio. Las representaciones audiovisuales e interactivas del cáncer en los videojuegos. Interface Comunicação, Saúde, Educação, 23. https://doi.org/10.1590/interface.180363

González Requena, J. (1988). El Discurso televisivo : espectáculo de la posmodernidad. Cátedra.

González Requena, J. (2006). Clásico, manierista, postclásico. Los modos del relato en el cine de Hollywood. Castilla Ediciones.

Grondin, J. (2018). Del sentido de las cosas: La idea de la metafísica. Herder.

Heidegger, M. (2009). Sery tiempo. Trotta.

Hidalgo Marí, T., \& Sánchez Olmos, C. (2015). Las series españolas en You'Tube: comparativa de género sobre el fenómeno fan. Miguel Hernández. Communication Journal, 6. https://doi. org/10.21134/mhcj.v0i6.88

Lacan, J. (1981). El seminario 20: Aun. Paidós. 
Lacan, J. (1984). El Seminario 3: Las psicosis. Paidós.

Loriguillo-López, A., \& Sorolla-Romero, T. (2015). "Vive, muere, repite": El blockbuster ante el desafío interactivo. Fonseca, Journal of Communication, 11, 118-132.

Marcos Molano, M., Romero Chamorro, S. F., \& Santorum González, M. (2019). El Storyworld en la narrativa de los Juegos Documentales Interactivos: Revista ICONO14 Revista Científica de Comunicación y Tecnologías Emergentes, 17(1), 39-59. https://doi.org/10.7195/ri14.v17i1.1246

Martín Núñez, M. (2011). Tensiones entre los viejos y los nuevos medios: una aproximación al análisis del videojuego como discurso audiovisual. La Comunicación Pública, Secuestrada Por El Mercado, 1-14.

Martín Núñez, M., \& García Catalán, S. (2015). Un segundo más y los caciques desaparecerían: El flash político de julián barón. Fotocinema: Revista Científica de Cine y Fotografía, 10, 327-351.

Marzal Felici, J. J., \& Gómez Tarín, F. J. (2015). Diccionario de conceptos y términos audiovisuales. Cátedra.

Ménard, A. D., Weaver, A., \& Cabrera, C. (2019). "There are Certain Rules that One Must Abide by": Predictors of Mortality in Slasher Films. Sexuality and Culture, 23(2), 621-640. https://doi. org/10.1007/s12119-018-09583-2

Navarro Remesal, V. (2016). Libertad dirigida: Una gramática del análisis y diseño de videojuegos. Shangrila. $\quad$ http://www.tdx.cat/bitstream/handle/10803/111168/tesi. pdf;jsessionid=18577A8F96CA3B0FE387197D7BE75CEF.tdx1? sequence=1

Navarro Remesal, V. (2019). Ser todo, ser nada: La subjetividad en el videojuego más allá del avatar. Tropelías, 31, 156-173.

Palao Errando, J. A., Loriguillo López, A., \& Sorolla Romero, T. (2018). Beyond the Screen, Beyond the Story: The Rhetorical Battery of Post-Classical Films. Quarterly Review of Film and Video, 35(3), 224-245. https://doi.org/10.1080/10509208.2017.1409097

Parrondo, E. (2014). Fantasía y realidad en "Pa negre” (Agustí Villaronga, 2010). Trama \& Fondo: Revista de Cultura, 37.

Pérez Latorre, Ó. (2012). El Lenguaje videolúdico: análisis de la significación del videojuego. Lartes.

Pérez-Latorre, Ó., Oliva, M., \& Besalú, R. (2017). Videogame analysis: a social-semiotic approach. Social Semiotics, 27(5), 586-603. https://doi.org/10.1080/10350330.2016.1191146

Petridis, S. (2019). Anatomy of the Slasher Film: A Theoretical Analysis. McFarland \& Company.

Planells, A. J. (2015). Videojuegos y mundos de ficción. Cátedra.

Rodríguez Serrano, A., Martín-Núñez, M., \& Gil-Soldevila, S. (2017). Ludologic design and augmented reality. The game experience in Pokémon Go! (Niantic, 2016). Revista Latina de 
Comunicacion Social, 72. https://doi.org/10.4185/RLCS-2017-1185

Rodríguez Serrano, Aarón. (2014). Cuando los videojuegos escribieron el Holocausto: Análisis de Wolfenstein: The New Order (Machinegames, 2014). Historia y Comunicación Social, 19, 193207.

Siabra Fraile, J. A. (2012). Bosquejo de una metafísica del videojuego. Círculo Rojo.

\section{Ludografía}

Blizzard (Desarrolladora) (2005). World of Warcraft [Videojuego]. Estados Unidos.

Cage, David (2018). Detroit: Become Human [Videojuego]. París: Quantic Dreams.

Galactic Café (Desarrolladora) (2013). The Stanley Parable [Videojuego]. Estados Unidos.

Giant Sparrow (Desarrolladora) (2017). What Remains of Edith Finch? [Videojuego]. Estados Unidos.

Hello Games (Desarrolladora) (2016). No man's sky [Videojuego]. Reino Unido.

Kojima, Hideo (2019). Death Stranding [Videojuego]. Japón: Kojima Productions.

Nintendo (2018) (Desarrolladora). Pokemon Let's Go Pikachu! [Videojuego]. Japón

Respawn Entertainment (Desarrolladora) (2019). Star Wars: Jedi Fallen Order [Videojuego]. Estados Unidos

Sega (Desarrolladora) (1986). Alex Kidd in Miracle World [Videojuego]. Japón.

Sony (Desarrolladora) (2017). WipEout Omega [Videojuego]. Japón.

Supermassive Games (Desarrolladora) (2015). Until Dawn [Videojuego]. Reino Unido.

Supermassive Games (Desarrolladora) (2017). Intenciones ocultas [Videojuego]. Reino Unido.

Supermassive Games (Desarrolladora) (2018). Man of Medan [Videojuego]. Reino Unido.

\section{Filmografía}

Alfred Hitchcock presents... (CBS, 1952-1965).

Are you Afraid of the Dark? (YTV, 1990-1995).

Creepshow (George A. Romero, 1982).

Tales from the Crypt (HBO, 1989-1996). 
MHCJ Vol. 11 (2) | Año 2020- Artículo no 8 (159) - Páginas 163 a 180 - mhjournal.org

\section{(c) (†) ()}

Licencia Creative Commons

Miguel Hernández Communication Journal

mhjournal.org

\section{Cómo citar este texto:}

Aarón Rodríguez Serrano (2020): Tiempo de jugar, tiempo de morir: Apuntes sobre Until Dawn y Man of Medan (Supermassive Games, 2015 y 2019), en Miguel Hernández Communication Journal, Vol. 11 (2), pp. 163 a 180. Universidad Miguel Hernández, UMH (Elche-Alicante). DOI: http://dx.doi.org/10.21134/mhcj. v11i0.335 